\title{
Pinning Force Scaling Analysis of Polycrystalline $\mathrm{MgB}_{2}$
}

\author{
M. R. Koblischka ${ }^{1,2}$ (D) $\cdot$ A. Wiederhold ${ }^{1} \cdot$ A. Koblischka-Veneva ${ }^{1,2} \cdot$ C. Chang $^{3}$
}

Received: 30 November 2019 / Accepted: 8 February 2020 / Published online: 21 July 2020

(c) The Author(s) 2020

\begin{abstract}
Flux pinning force scaling $f=F_{p} / F_{p \text {,max }}$ vs. $h=H_{a} / H_{\text {irr }}$ was performed on a variety of pure $\mathrm{MgB}_{2}$ samples, including a spark plasma sintered (SPS) one and a series of samples sintered at various reaction temperatures ranging between 775 and $950{ }^{\circ} \mathrm{C}$. The SPS sample exhibits a well-developed scaling at all temperatures, and also the sintered samples prepared at $950{ }^{\circ} \mathrm{C}$; however, the obtained peak positions of the pinning force scalings are distinctly different: The SPS sample reveals dominating pinning at grain boundaries, whereas the dominating pinning for the other one is point-pinning. All other samples studied reveal an apparent non-scaling of the pinning forces. The obtained pinning parameters are discussed in the framework of the Dew-Hughes' pinning force scaling approach.
\end{abstract}

Keywords $\mathrm{MgB}_{2} \cdot$ Flux pinning forces $\cdot$ Scaling of flux pinning forces $\cdot$ Grain boundary pinning $\cdot$ Core pinning

\section{Introduction}

The $\mathrm{MgB}_{2}$ superconductors are interesting for various applications as they offer the metallic character which enables a simple processing route and no involved rare earth materials, which reduces the costs involved [1, 2]. The modern cryocooling techniques enable a temperature of $20 \mathrm{~K}$ to be reached, which is commonly accepted as the optimum one for applications of $\mathrm{MgB}_{2}$. Besides these advantages, the still limited critical current densities and the presence of flux jumps require further studies on the acting flux pinning mechanism(s) in $\mathrm{MgB}_{2}$ superconductors [3-10].

The pinning force scaling as introduced by Kramer [11] and Dew-Hughes (DH) [12] is an important tool to study the pinning mechanisms acting in a given superconductor sample. This applies not only for the conventional metallic superconductors, where the pioneering work was performed

M. R. Koblischka

miko@shibaura-it.ac.jp; m.koblischka@gmail.com

1 Experimental Physics, Saarland University, P.O.Box 151150, 66041, Saarbrücken, Germany

2 Present address: Superconducting Materials Laboratory, Department of Materials Science and Engineering, Shibaura Institute of Technology, Tokyo, 135-8548, Japan

3 Institut Jean Lamour, UMR CNRS-Université de Lorraine, 54506, Vandœvre-lès-Nancy, France on, but also for the high- $T_{c}$ counterparts [13-16] as well as $\mathrm{MgB}_{2}$ [16-20] and the iron-based superconductors [21]. The main difference is given by the use of the irreversibility field, $H_{\text {irr }}$ instead of the upper critical field, $H_{\mathrm{c} 2}$ for the scaling, as $H_{\text {irr }}$ determines the upper limit of the flux pinning (= irreversible region in the H-T diagram). The flux pinning can be obtained from the scaled pinning force data $f=F_{p} / F_{p, \max }$ vs. $h=H_{a} / H_{\text {irr }}$ by a fit to the functional dependence given by the following:

$f=A(h)^{p}(1-h)^{q}$.

The resulting parameters, $p$ and $q$, determine the dimensionality and the type of the dominating flux pinning, whereas the numerical parameter $A$ in (1) is obtained from the normalization condition $F_{p} / F_{p, \max }=1$. The corresponding peak position, $h_{0}$, can be calculated as $h_{0}=$ $\frac{p}{p+q}$.

In the model of $\mathrm{DH}$, there are six pinning functions $f(h)$ describing the core pinning using (1): (1) $p=0, q=2$ : normal, volume pinning; (2) $p=1, q=1: \Delta \kappa$-pinning, volume pins; (3) $p=1 / 2, q=2$ : normal, surface pins; (4) $p=3 / 2, q=1: \Delta \kappa$-pinning, surface pins; (5) $p=1, q=2$ : normal, point pins; and (6) $p=2, q=1: \Delta \kappa$-pinning, point pins. Furthermore, the function (3) is predicted by Kramer [11] for shear-breaking in the case of a set of planar pins. A seventh function describing the magnetic interaction can be disregarded in the present case. Therefore, the parameter $p$ can assume the values $0,0.5,1,1.5$, and 2 , which describes the dimensionality of the pins, and the parameter $q$ can only 
take the values 1 and 2, describing the type of pinning (i.e., normal conducting or superconducting).

Having this in mind, the analysis of the pinning force scaling provides important information on the flux pinning properties of a given sample, provided that the $F_{p}$ data exhibit a proper scaling. To obtain information on the flux pinning in $\mathrm{MgB}_{2}$ samples, we therefore investigated a pure SPS-produced $\mathrm{MgB}_{2}$ sample with its high density close to the ideal value, and several members of a series of sintered, polycrystalline $\mathrm{MgB}_{2}$ samples prepared at various reaction temperatures ranging between 775 and $950{ }^{\circ} \mathrm{C}$. As an interesting result, some of the samples exhibit a welldeveloped scaling, while others do not. The results obtained are discussed in the framework of the DH pinning model.

\section{Experimental Procedures}

The sample preparation steps for the SPS sample are described in Ref. [22, 23], and the fabrication of the polycrystalline, sintered samples is discussed in Ref. [24]. All samples were pure samples without any additions. The $\mathrm{X}$-ray analysis and electron backscatter diffraction (EBSD) investigations revealed the presence of small amounts of $\mathrm{MgO}$ and $\mathrm{MgB}_{4}$ particles in the samples prepared at reaction temperatures above $850^{\circ} \mathrm{C}$. The $\mathrm{MgB}_{4}$ particles in the SPS sample were found to be located to a large extend along the grain boundaries, and not within the $\mathrm{MgB}_{2}$ grains, so these particles cannot provide additional flux pinning [25]. This is important as the goal of this contribution is to study the basic flux pinning properties of $\mathrm{MgB}_{2}$, which would otherwise be overlaid with the strong pinning provided by the secondary particles embedded in the $\mathrm{MgB}_{2}$ matrix. A thorough magnetic characterization of the SPS sample was presented by Jirsa et al. [26]. The superconducting properties of the samples studied are summarized in Table 1 below, presenting the maximum pinning force, $F_{p \text {, max }}$, the current density at $20 \mathrm{~K}, j_{c}(20 \mathrm{~K})$, in self-field condition, and trapped fields obtained at $20 \mathrm{~K}$ on samples with a 20 $\mathrm{mm}$ diameter and 5- or 7-mm thickness, respectively. The samples were state-of-the-art for pure samples, which is revealed by trapped field experiments on two stacked SPS samples with a 20-mm diameter (10-mm thickness), where a trapped field of $4.66 \mathrm{~T}$ could be recorded at $20 \mathrm{~K}(6.25 \mathrm{~T}$ at $10 \mathrm{~K})$ [27].

For the magnetic measurements, small samples $(2 \times$ $2 \times 1.5 \mathrm{~mm}^{3}$ ) were cut from the big pellets. The magnetic measurements were performed using a Quantum Design MPMS3 SQUID system with a $\pm 7 \mathrm{~T}$ applied field, and Quantum Design PPMS systems equipped either with an extraction magnetometer or with VSM option ( $\pm 7 \mathrm{~T}$ and $\pm 9 \mathrm{~T}$ applied magnetic field). In all cases, the field was applied perpendicular to the sample surface. The field sweep rate was always $0.36 \mathrm{~T} / \mathrm{min}$. The temperatures selected for the investigation were between 5 and $35 \mathrm{~K}$ in steps of $5 \mathrm{~K}$. From the magnetization data, the critical current densities, $j_{c}$, were evaluated using the extended Bean approach for rectangular samples [28]. The irreversibility lines, $H_{\text {irr }}(H, T)$, were determined using a criterion of $100 \mathrm{~A} / \mathrm{cm}^{2}$.

\section{Results and Discussion}

In all samples, we had problems with flux jumps at the low temperatures, $T=5 \mathrm{~K}$ and $10 \mathrm{~K}$. Therefore, these measurements were repeated several times to allow a reconstruction of the enveloping curve(s), which were then used for the pinning force calculation.

Figure 1a-c present the pinning force scaling of samples S800 (a), S850 (b), and SPS (c). The final pinning function according to (1) is obtained from a fit to all data and is indicated by a solid, red line. The pinning force scalings of samples S775 and S950 were already shown in Ref. [19], and the scaling behavior of sample S775 is similar to that of sample S800 shown here (a). The main difference between
Table 1 Scaling parameters $A$, $p, q$ and $h_{0}$ for all $\mathrm{MgB}_{2}$ samples studied here

\begin{tabular}{llllllll}
\hline $\begin{array}{l}\text { Sample name } \\
T_{\text {reaction }\left({ }^{\circ} \mathrm{C}\right)}\end{array}$ & $A$ & $p$ & $q$ & $h_{0}$ & $\begin{array}{l}F_{p, \max } \\
\left(10^{6} \mathrm{~N} / \mathrm{m}^{3}\right)\end{array}$ & $\begin{array}{l}j_{c}(20 \mathrm{~K}) \\
\left(10^{3} \mathrm{~A} / \mathrm{cm}^{2}\right)\end{array}$ & $\begin{array}{l}\text { Trapped fields } \\
(\mathrm{T})(20 \mathrm{~K})\end{array}$ \\
\hline S775 & 2.59 & 0.43 & 1.20 & 0.26 & 67.8 & 176 & 1.5 \\
S800 & 2.13 & 0.34 & 0.96 & 0.26 & 68.2 & 180 & 1.42 \\
S805 & 4.31 & 0.70 & 1.75 & 0.29 & 110 & 185 & 1.50 \\
S850 & 3.05 & 0.57 & 1.21 & 0.33 & 57.1 & 128 & 1.32 \\
S950 & 4.20 & 0.66 & 1.78 & 0.32 & 65.6 & 135 & 1.25 \\
SPS & 5.12 & 0.83 & 1.77 & 0.21 & 231 & 300 & 1.45 \\
\hline
\end{tabular}

The data shown stem from fits to all temperatures measured of a given sample. The maximum pinning forces, $F_{p \text {, max }}$, are the data of the maximal measured values, $F_{p}=j_{c} \times B$ at $T=10 \mathrm{~K}$, and $j_{c}(20 \mathrm{~K})$ was measured at self-field. The trapped field values were obtained at $T=20 \mathrm{~K}$ for samples with a 20 -mm diameter and 7-mm thickness [24], whereas the sample SPS had only a thickness of $5 \mathrm{~mm}$ [23] 


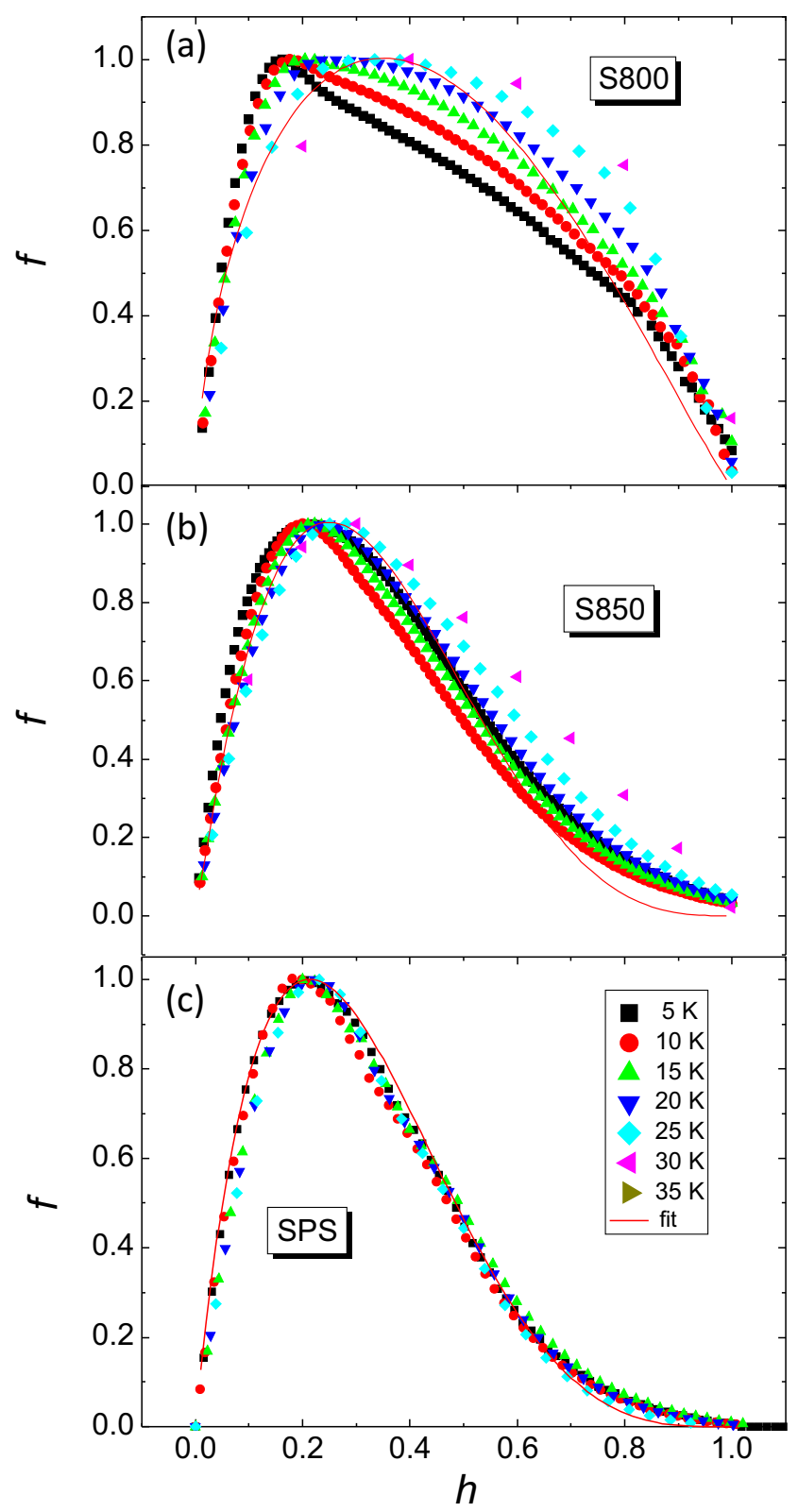

Fig. 1 a Pinning force scaling of sample S800. b Pinning force scaling of sample S950. c Pinning force scaling of sample SPS. The fits to all data according to (1) are indicated by red lines; the obtained fit parameters are listed in Table 1

the two scalings is the more pronounced peak in $F_{p}$ at the low temperatures, the origin of which is discussed in another publication [29]. Note the obvious non-scaling of the data of sample S800, which reveal a clear shift of the peak position and a strengthened high- $h$ part when increasing the temperature. The scalings of samples S850 (b) and S950 are also found to be quite similar to each other. As one can see from the fit functions obtained as shown in Fig. 2 below, the SPS sample (c) reveals in contrast a well-developed scaling behavior.
Figure 2 presents the fit functions obtained for all samples investigated; the corresponding fitting parameters are given in Table 1 . Here, we see that the SPS sample shows a clearly different scaling with steep increase at low fields, a peak position, $h_{0}=0.21$, and the typical tail at high fields. All these features are typical for flux pinning provided by the grain boundaries (DH function (3)). The samples S850 and S950 show a less steep increase at low fields, and as consequence, a peak at $h_{0}=0.32 / 0.33$. This is a strong indication for flux pinning provided by small, non-superconducting defects (DH pinning function (5)). The other samples show an intermediate behavior, but it is important to mention here that the scaling fits of these samples have a much larger deviation as the ones mentioned before. So, one has to admit that these samples do not show a proper pinning force scaling. The main result from the pinning force scaling is that in the sintered samples, flux pinning at GBs is important at lower temperatures, whereas pinning at the small, normal-conducting inclusions is dominating the high field, and high temperature range.

Regarding the values of the scaling parameter $p$ obtained from the fits (see Table 1), we find that all data are ranging in the span of the possible DH values, and the same applies to the $q$ values. Thus, the flux pinning properties of the pure $\mathrm{MgB}_{2}$ samples fit into the scope of the DH approach, which was developed for metallic superconductors. In contrast, on various high- $T_{c}$ samples, much higher $p$ or $q$ values were found, which reflect a different type of vortex-pin interaction. Furthermore, Table 1 gives the data for the maxima of the pinning forces, $F_{p, \max }$, measured via $F_{p}=$ $j_{c} \times B$ at a temperature of $10 \mathrm{~K}$. The sample S800 shows slightly higher $F_{p \text {,max }}$ as sample $\mathrm{S} 775$, and sample $\mathrm{S} 805$ has the highest value of all sintered $\mathrm{MgB}_{2}$ samples. The sample S850 exhibits the lowest $F_{p \text {,max }}$, and sample S950 reaches

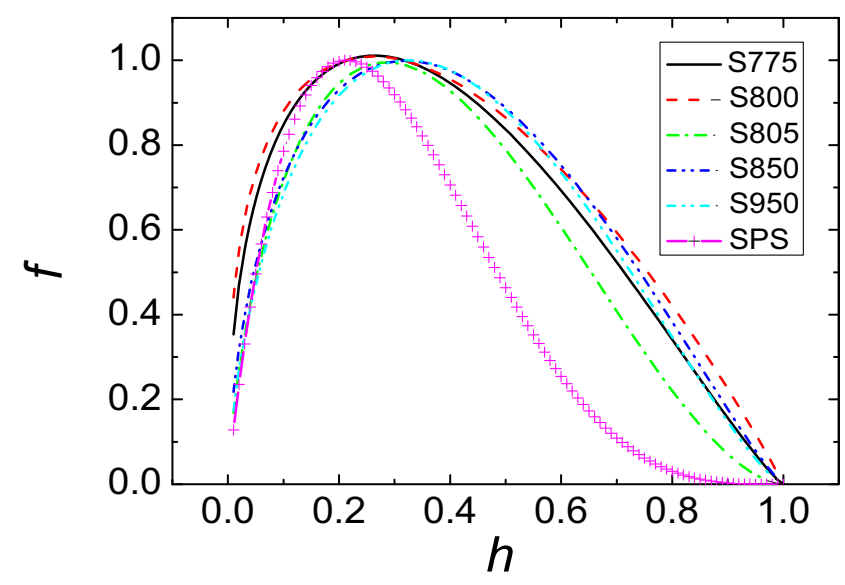

Fig. 2 Summary of all pinning force scalings. Plotted are the fit functions to all data of a sample 
again a similar value as the samples S775/S800. In contrast, the SPS sample shows the highest $F_{p \text {,max }}$ measured here.

This non-scaling of the pinning force data may have different reasons. First of all, this non-scaling was observed already by various authors. In Ref. [16], it was speculated that this is due to strongly misoriented $\mathrm{MgB}_{2}$ grains with the boron plane oriented perpendicular to the applied field. If the field is too strong, these grains would render normal and thus be able to provide more flux pinning according to pinning function (5). As the grains in polycrystalline samples are assumed to be randomly oriented, this is a gradual process according to the crystallographic orientations of the $\mathrm{MgB}_{2}$ grains. For $\mathrm{MgB}_{2}$, the anisotropy values $\gamma$ range in the literature between 1 and 7 [30], so the effect could be a pronounced one. However, our EBSD investigations of the sintered, polycrystalline samples [31] have shown that the $\mathrm{MgB}_{2}$ grains are not only fully randomly oriented but also have some distinct orientations and practically no fully misoriented grains with a misorientation of $90^{\circ}$. In Ref. [19], we had plotted the temperature dependence of the anisotropy parameter according to Ref. [30] together with the $h_{0}(T)$ data, and the outcome shows that the anisotropy reduces on increasing temperature. Therefore, this explanation for the non-scaling behavior cannot account for the apparent non-scaling.

The strength of the DH scaling analysis is the proper scaling behavior of the pinning forces over a wide temperature range. The so obtained scaling behavior then makes any conclusion about the acting flux pinning mechanism much stronger as if one would analyze the data of only one temperature. However, the present nonscaling of the polycrystalline $\mathrm{MgB}_{2}$ samples require a different approach to elucidate the ongoing changes with temperature.

As the pinning force scaling of the polycrystalline, sintered $\mathrm{MgB}_{2}$ samples reveals an apparent non-scaling, we performed the fits of the pinning functions to each measured temperature separately. Although this is, in principle, against the idea of the $\mathrm{DH}$ approach, this allows us to get information how the changes of pinning progress when raising the temperature. The results of this procedure are illustrated as the temperature dependence of the peak position, $h_{0}$ (Fig. 3) and the temperature dependence of the pinning parameters $p$ and $q$ (Fig. 4a, b) below.

The peak positions, $h_{0}$, as function of temperature (Fig. 3) are practically flat for the SPS sample as it is expected from a good scaling at all temperatures. All other samples reveal an increasing $h_{0}$ on increase of the temperature. The samples S850 and S950, which show a still acceptable scaling, also exhibit an increase of $h_{0}$, but in a limited range only, thus justifying the scaling approach. The other samples reveal a large variation of $h_{0}(T)$. To compare the temperature-dependent $h_{0}$ data with those of the overall

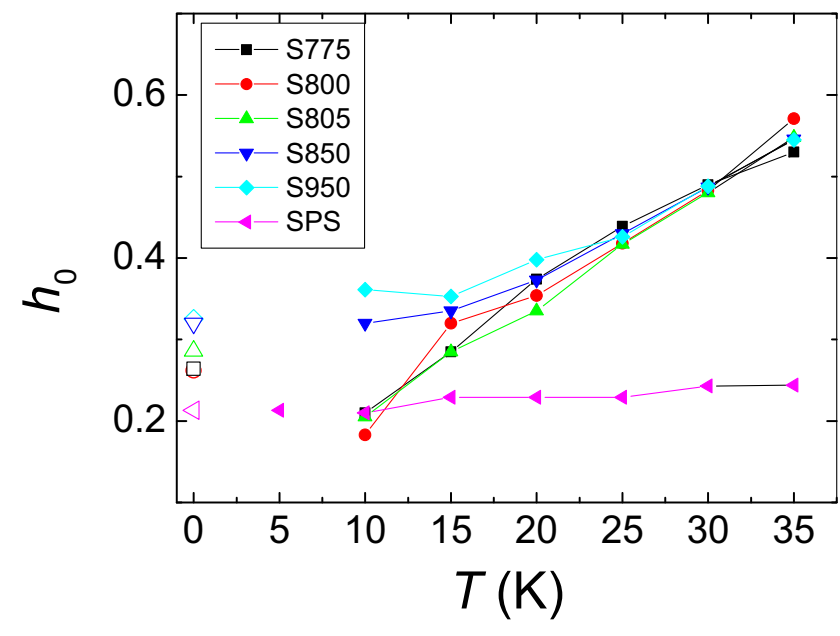

Fig. 3 The peak positions, $h_{0}$, as determined from the $F_{p}$ scalings of each temperature alone. The open symbols plotted at $T=0 \mathrm{~K}$ give the $h_{0}$ values for the common scalings as determined from Fig. 2 for comparison. The lines drawn are guides to the eye

scaling as presented in Fig. 2, we have plotted the resulting peak positions at $T=0$ using open symbols, but in the same colors. The comparison of these peak positions shows

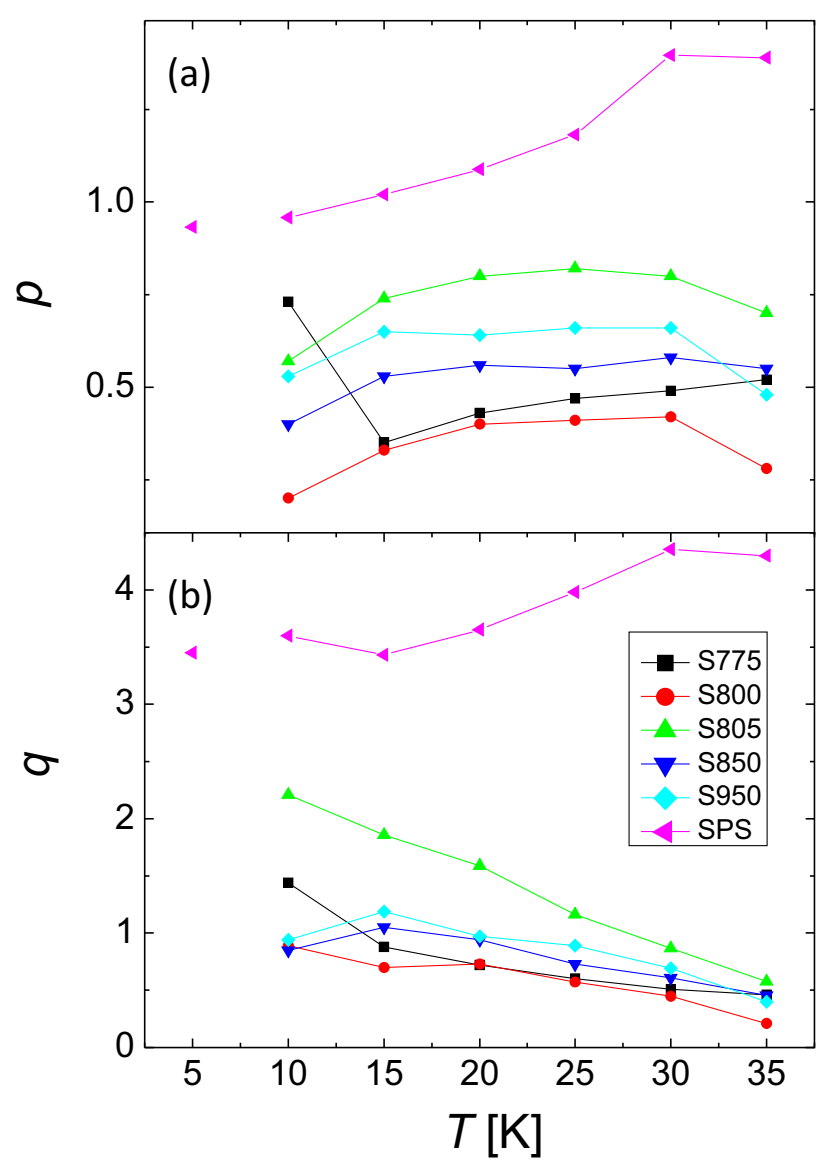

Fig. 4 The variation of the parameters $p$ (a) and $q$ (b) as a function of temperature using the $\mathrm{DH}$ model for each temperature. The lines drawn are guides to the eye 
a clear tendency of the overall fits to smooth out the steep increase of $h_{0}$ as there are less and less data points at the elevated temperatures.

Figure $4 \mathrm{a}$ and $\mathrm{b}$ present the temperature dependence of the scaling parameters $p$ and $q$ as obtained from the individual fittings to each measured temperature. First of all, one should note that in the original DH scaling, $p$ can take the values of $0,0.5,1,1.5$, and 2 (dimensionality and pinning character), and the parameter $q$ can only take the values 1 and 2 (pinning character). Regarding now Fig. 4a, we see that this is not the case for the present data. The data for $p$ range between 0.3 and 1.7, so still in the range of the DH model. The situation is different for the $q$ values, which are around 1 for low $T$, but then tend clearly down to values around 0.5 . The two samples with stronger pinning show high values for $q$ at low $T$ (S805 at $\sim 2$ and SPS at 3.5), but then the data for sample S805 decrease towards 0.7, whereas the values for the SPS sample even reach $q=4$. Such high values for $q$ are not uncommon; the pinning force scalings of most high $T_{c}$ superconductors also exhbit $q>2$ (see, e.g., [13]).

The pinning force scaling data now suggest another explanation of what is going on in the samples at elevated temperatures. In a previous publication, we discussed the origin of the sharp, narrow peak in the pinning force vs. field diagrams. The reason that this pinning force peak vanishes at higher fields and temperatures was found in the decreasing grain connectivity, which is essential to maintain the current flow through the entire sample. As consequence, this may also take place in our case-at higher magnetic fields, the currents flowing in the samples may circle through smaller entities as the current flow through the entire sample perimeter breaks down after having reached the peak in $F_{p}$. The pores being present in the sintered, polycrystalline $\mathrm{MgB}_{2}$ samples also contribute to this effect, forcing the currents on percolative paths.

The most extreme case of this breakdown of the current flow was observed by Dou et al. [32] on compacted and sintered $\mathrm{MgB}_{2}$ samples with grains of a $200-\mu \mathrm{m}$ size. Here, it was necessary to calculate the current density from the sample perimeter at low fields, and at high fields, the grain size was used instead. In this way, the authors could reconstruct a relatively smooth $j_{c}(H)$ curve. The appearance of the clear distinction of the two regimes was already seen in the $M(H)$ plots, and the relatively big grain size may be the reason for this observation. The present $\mathrm{MgB}_{2}$ samples have much smaller grains in the 100-500nm range, so the $M(H)$ loops of all our samples appear fully regular in shape. However, if we presume a change of the current flow radius in the samples, this leads in consequence to a non-scaling behavior of the pinning force data obtained.

Finally, the pinning force scaling as modified by Eisterer [33] is applied to the present data. This approach modifies the DH scaling by using a different scaling field $h=H / H_{n}$ instead of $H_{\text {irr }}$. Here, $H_{n}$ denotes the magnetic field, which is reached at $50 \%$ above the maximum pinning force. This forces all curves through the point $(0.5,1)$ in the scaling diagram. As a result, the area below $h_{n}=1$ is less sensitive to anisotropy and percolation, and the shift of the peak due to this is negligible. If the value of $\gamma$ changes for example between 2 and 5, the peak shift only causes a deviation of $3 \%$ for pinning at grain boundaries.

The fits for this scaling were calculated using the formula as follows:

$F_{p} / F_{\mathrm{p}, \max }=A\left(\frac{H}{H_{n}}\right)^{p}\left(2-\left(\frac{H}{H_{n}}\right)^{q}\right)$,

and the resulting temperature dependence of the fit parameters $p$ and $q$ is displayed in Fig. 5a, b.

We have already used the Eisterer scaling to discuss the flux pinning proprties in Ref. [19] and also for carbonadded, polycrystalline $\mathrm{MgB}_{2}$ samples [34]. The advantage of this approach is to take care for the anisotropy and percolation, and thus peak positions smaller than 0.22 can be explained, and the values for $h_{0}$ are shifted as compared

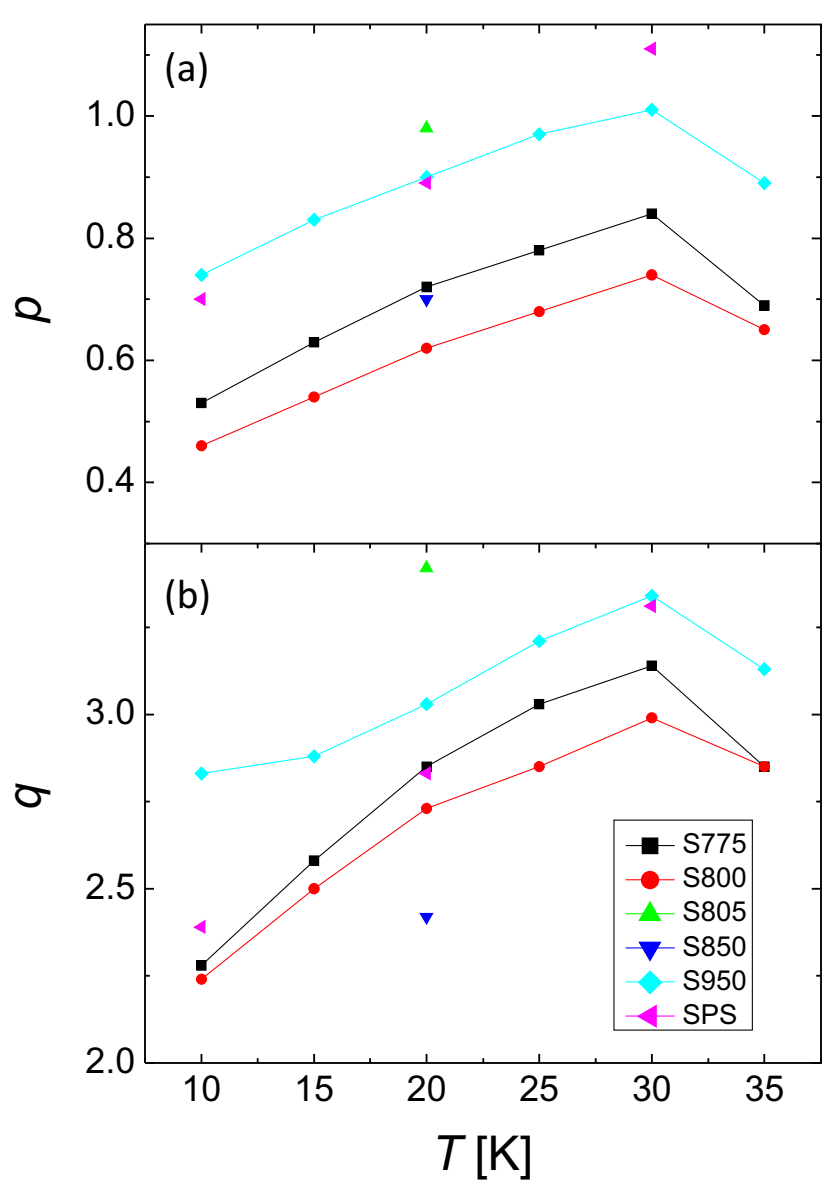

Fig. 5 The variation of the parameters $p$ (a) and $q$ (b) in the Eisterer scaling. The lines drawn are guides to the eye 
to the DH ones. In the present case, the apparent nonscaling of the samples S775, S800, and S805 prevails. The resulting fit parameters $p$ and $q$ exhibit a clear temperature dependence as well. In contrast to the original DH model, the behavior of the parameters $p$ and $q$ shows a common tendency only, which is a drawback of the Eisterer approach. In contrast, nearly common values are found in the DH model when the flux pinning has a common origin. From the data presented here, it is clear that the percolation also changes with increasing temperature, which is another result pointing towards a change of the radius of the current flow. Furthermore, the main result of the DH scaling is also valid when regarding the behavior of the modified scaling of Eisterer.

All the present pinning force scalings demonstrate that the dominating flux pinning in the highly dense SPS sample is provided by the grain boundaries. The scaling works well in the entire temperature range, and the characteristic features of the obtained fitting function are reproduced by all data, even at the highest temperature of $T=35 \mathrm{~K}$ measured here. On the other side, the scalings of samples S850 and S950 are still acceptable ones, even though the peak positions exhibit a clear upturn on increasing temperature. Here, the pinning is dominantly provided by small, non-superconducting defects, which are highly likely represented by the $\mathrm{MgB}_{4}$ particles, being created in the samples due to the high reaction temperatures. Both types of scaling (DH and Eisterer) demonstrate the apparent nonscaling of the pinning forces of all other samples studied here. The scalings reveal that for low temperatures, the GBs are the responsible pinning mechanism which changes gradually to pinning at small, normal-conducting inclusions, and even higher peak positions as 0.33 can be obtained, especially at high temperatures. The change of the peak position is further always accompanied by an entire change of the shape of the pinning function. All this leads to the conclusion that there is a change of the current flow radius at elevated temperatures, being due to a gradual decoupling of the $\mathrm{MgB}_{2}$ grains. This observation has, therefore, important consequences for the development of $\mathrm{MgB}_{2}$-based superconducting permanent magnets [35-38] with higher trapped fields.

\section{Conclusions}

To conclude, we performed flux pinning force scalings on a SPS- $\mathrm{MgB}_{2}$ sample and a series of sintered, polycrystalline $\mathrm{MgB}_{2}$ samples prepared at various reaction temperatures. The scaling works very well on the SPS sample, indicating a dominant pinning provided by grain boundaries. For the samples sintered at high reaction temperatures, S850 and S950, a still acceptable scaling is obtained which indicates a dominant pinning provided by small, non-superconducting defects. All other samples studied here show an apparent non-scaling, as the peak in $f$ shifts toward higher values on increasing the temperature. Scaling using the Eisterer approach does not provide new insights, but confirms the conclusions drawn from the DH scaling. Thus, the data support the conclusion that at elevated temperature, the $\mathrm{MgB}_{2}$ grains decouple, and consequently, the current flow in the sample is changed, leading to the apparent nonscaling of the flux pinning forces in some of the samples.

Acknowledgments We thank J. Noudem (CRISMAT, Caen, France) for the SPS sample, and K. Inoue, M. Muralidhar (SIT, Tokyo, Japan) for the sintered $\mathrm{MgB}_{2}$ samples. Valuable discussions with $\mathrm{K}$. Berger, Q. Nouailhetas, and B. Douine (GREEN, Université de Lorraine, Nancy, France) concerning the trapped field measurements on $\mathrm{MgB}_{2}$ samples are acknowledged.

Funding Information Open Access funding provided by Projekt DEAL. This work is part of the SUPERFOAM international project funded by ANR and DFG under the references ANR-17-CE05-0030 and DFG-ANR Ko2323-10, respectively.

Open Access This article is licensed under a Creative Commons Attribution 4.0 International License, which permits use, sharing, adaptation, distribution and reproduction in any medium or format, as long as you give appropriate credit to the original author(s) and the source, provide a link to the Creative Commons licence, and indicate if changes were made. The images or other third party material in this article are included in the article's Creative Commons licence, unless indicated otherwise in a credit line to the material. If material is not included in the article's Creative Commons licence and your intended use is not permitted by statutory regulation or exceeds the permitted use, you will need to obtain permission directly from the copyright holder. To view a copy of this licence, visit http:// creativecommonshorg/licenses/by/4.0/.

\section{References}

1. Eisterer, M.: Magnetic properties, and critical currents of $\mathrm{MgB}_{2}$. Supercond. Sci Technol. 20, R47-R74 (2007)

2. Tomsic, M., Rindfleisch, M., Yue, J., McFadden, K., Phillips, J., Sumption, M.D., Bhatia, M., Bohnenstiehl, S., Collings, E.W.: Overview of $\mathrm{MgB}_{2}$ superconductor applications. Int. J. Appl. Ceram. Technol. 4, 250-259 (2007)

3. Dou, S.X., Soltanian, S., Horvat, J., Wang, X.L., Zhou, S.H., Ionescu, M., Liu, H.K., Munroe, P., Tomsic, M.: Enhancement of the critical current density and flux pinning of $\mathrm{MgB}_{2}$ superconductor by nanoparticle doping. Appl. Phys. Lett. 81, 3419-3421 (2002)

4. Yamamoto, A., Shimoyama, J., Ueda, S., Katsura, Y., Horii, S., Kishio, K.: Improved critical current properties observed in $\mathrm{MgB}_{2}$ bulks synthesized by low-temperature solid-state reaction. Supercond. Sci. Technol. 18, 116-121 (2005)

5. Giunchi, G., Ripamonti, G., Cavallin, T., Bassani, E.: The reactive liquid $\mathrm{Mg}$ infiltration process to produce large superconducting bulk $\mathrm{MgB}_{2}$ manufacts. Cryogenics 46, 237-242 (2006)

6. Mikheenko, P., Martinez, E., Bevan, A., Abell, J.S., MacManusDriscoll, J.: Grain boundaries and pinning in bulk $\mathrm{MgB}_{2}$. Supercond. Sci. Technol. 20, S264-S270 (2007) 
7. Martinez, E., Martinez-Lopez, M., Millan, A., Mikheenko, P., Bevan, A., Abell, J.S.: Temperature and magnetic field dependence of the $n$-values of $\mathrm{MgB}_{2}$ superconductors. IEEE Trans. Appl. Supercond. 17, 2738-2741 (2007)

8. Martinez, E., Mikheenko, P., Martinez-Lopez, M., Millan, A., Bevan, A., Abell, J.S.: Flux pinning force in bulk $\mathrm{MgB}_{2}$ with variable grain size. Phys. Rev. B 75, 134515 (2007)

9. Cheng, C.H., Sorrell, C.: Defect control at nanoscale and flux pinning enhancement in $\mathrm{MgB}_{2}$ superconductor. J. Modern Trans. 20, 65-69 (2012)

10. Naito, T., Sasaki, T., Fujishiro, H.: Trapped magnetic field and vortex pinning properties of $\mathrm{MgB}_{2}$ superconducting bulk fabricated by a capsule method. Supercond. Sci. Technol. 25, 095012 (2012)

11. Kramer, E.J.: Scaling laws for flux pinning in hard superconductors. J. Appl. Phys. 44, 1360-1370 (1973)

12. Dew-Hughes, D.: Flux pinning mechanisms in type-II superconductors. Philos. Mag. 30, 293-305 (1974)

13. Koblischka, M.R., van Dalen, A.J.J., Higuchi, T., Yoo, S.I., Murakami, M.: Analysis of pinning in $\mathrm{NdBa}_{2} \mathrm{Cu}_{3} \mathrm{O}_{7-\delta}$ superconductors. Phys. Rev. B 58, 2863 (1998)

14. Koblischka, M.R.: Pinning forces and scaling in high- $T_{c}$ superconductors. Phys. C 282-287, 2193-2194 (1997)

15. Koblischka, M.R.: Pinning in bulk high- $T_{c}$ superconductors. Inst. Phys. Conf. Ser. 58, 1141-1144 (1997)

16. Sandu, V.: Pinning force scaling and its limitation in intermediate and high-temperature superconductors. Mod. Phys. Lett B 26, 1230007 (2012)

17. Sandu, V., Chee, C.h.J.: On the scaling law of the pinning force in $\mathrm{MgB}_{2}$ superconducting composites with magnetic nanoinclusions. J. Supercond. Nov. Magn. 26, 125-131 (2013)

18. Salem, A.F., Ziq, K.A., Bahgat, A.A.: Scaling behavior in aluminium doped $\mathrm{MgB}_{2}$ superconductor. J. Supercond. Nov. Magn. 26, 1517-1520 (2013)

19. Koblischka, M.R., Wiederhold, A., Muralidhar, M., Inoue, K., Hauet, T., Douine, B., Berger, K., Murakami, M., Hartmann, U.: Development of $\mathrm{MgB}_{2}$-based bulk supermagnets. IEEE Trans. Magn. 50, 9000504 (2014)

20. Badica, P., Aldica, G., Ionescu, A.M., Burdusel, M., Batalu, D.: The influence of different additives on $\mathrm{MgB} 2$ superconductor obtained by ex situ spark plasma sintering: pinning force aspects. In: Nishikawa, H., Iwata, N., Endo, T., Takamura, Y., Lee, G.H., Mele, P. (eds.) Correlated functional oxides: nanocomposites and heterostructures, pp. 75-116. Springer, Berlin (2017)

21. Koblischka, M.R., Muralidhar, M.: Pinning force scaling analysis of Fe-based high- $T_{c}$ superconductors. Int. J. Mod. Phys. B 30, 1630017 (2016)

22. Noudem, J.G., Aburras, M., Bernstein, P., Chaud, X., Muralidhar, M., Murakami, M.: Development in processing of $\mathrm{MgB}_{2}$ cryomagnet superconductors. J. Appl. Phys. 116, 163916 (2014)

23. Noudem, J.G., Dupont, L., Gozzelino, L., Bernstein, P.: Superconducting properties of $\mathrm{MgB}_{2}$ bulk shaped by spark plasma sintering. Mater. Today-Proc. 3, 545-549 (2016)

24. Muralidhar, M., Nozaki, K., Kobayashi, H., Zeng, X.L., Koblischka-Veneva, A., Koblischka, M.R., Inoue, K., Murakami, M.: Optimization of sintering conditions in bulk $\mathrm{MgB}_{2}$ material for improvement of critical current density. J. Alloys Compounds 649, 833-842 (2015)
25. Koblischka-Veneva, A., Koblischka, M.R., Schmauch, J., Noudem, J., Murakami, M.: Analysis of the microstructure of bulk $\mathrm{MgB}_{2}$ using TEM, EBSD and t-EBSD. J. Microsc. 274, 123-131 (2019)

26. Jirsa, M., Rames, M., Koblischka, M.R., Koblischka-Veneva, A., Berger, K., Douine, K.: Relaxation and pinning in spark-plasma sintered $\mathrm{MgB}_{2}$ superconductor. Supercond. Sci. Technol. 29, 025006 (2016)

27. Berger, K., Koblischka, M.R., Douine, B., Noudem, J., Bernstein, P., Hauet, T., Leveque, J.: High magnetic field generated by bulk $\mathrm{MgB}_{2}$ prepared by spark plasma sintering. IEEE Trans. Appl. Supercond. 29, 6801005 (2016)

28. Wiesinger, H.P., Sauerzopf, F.M., Weber, H.W.: On the calculation of $J_{c}$ from magnetization measurements on superconductors. Phys. C 203, 121-128 (1992)

29. Koblischka, M.R., Wiederhold, A., Koblischka-Veneva, A., Chang, C., Berger, K., Nouailhetas, Q., Douine, B., Murakami, M.: Origin of the sharp, low-field peak in $\mathrm{MgB}_{2}$ superconductors. submitted for publication. AIP Adv. 10, 015035 (2020)

30. Shi, Z.X., Tokunaga, M., Tamegai, T., Takano, Y., Togano, K., Kito, H., Ihara, H.: Out-of-plane and in-plane anisotropy of upper critical field in $\mathrm{MgB}_{2}$. Phys. Rev. B 68, 104513 (2003)

31. Koblischka-Veneva, A., Koblischka, M.R., Schmauch, J., Inoue, K., Muralidhar, M., Berger, K., Noudem, J.: EBSD analysis of $\mathrm{MgB}_{2}$ bulk superconductors. Supercond. Sci. Technol. 29, 044007 (2016)

32. Dou, S.X., Wang, X.L., Horvat, J., Milliken, D., Li, A.H., Konstantinov, K., Collings, E.W., Sumption, M.D., Liu, H.K.: Flux jumping and a bulk-to-granular transition in the magnetization of a compacted and sintered $\mathrm{MgB}_{2}$ superconductor. Phys. C 361, 79-83 (2001)

33. Eisterer, M.: Calculation of the volume pinning force in $\mathrm{MgB}_{2}$ superconductors. Phys. Rev. B 77, 144524 (2008)

34. Koblischka, M.R., Koblischka-Veneva, A., Muralidhar, M., Murakami, M.: Magnetic characterization of bulk C-added $\mathrm{MgB}_{2}$. IEEE Trans. Appl. Supercond. 29, 6800104 (2019)

35. Fuchs, G., Häßler, W., Nenkov, K., Scheiter, J., Perner, O., Handstein, A., Kanai, T., Schultz, L., Holzapfel, B.: High trapped fields in bulk $\mathrm{MgB}_{2}$ prepared by hot-pressing of ball-milled precursor powder. Supercond. Sci. Technol. 26, 122002 (2012)

36. Durrell, J.H., Dancer, C.E.J., Dennis, A., Shi, Y., Xu, Z., Campbell, A.M., Babu, N.H., Todd, R.I., Grovenor, C.R.M., Cardwell, D.A.: A trapped field of $>3 \mathrm{~T}$ in bulk $\mathrm{MgB}_{2}$ fabricated by uniaxial hot pressing. Supercond. Sci. Technol. 25, 112002 (2012)

37. Berger, K., Koblischka, M.R., Douine, B., Noudem, J., Bernstein, P., Hauet, T., Lévêque, J.: High magnetic field generated by bulk $\mathrm{MgB}_{2}$ prepared by spark plasma sintering. IEEE Trans. Appl. Supercond. 26, 6801005 (2016)

38. Miyazaki, T., Fukui, S., Ogawa, J., Sato, T., Oka, T., Scheiter, J., Häßler, W., Kulawansha, E., Yuanding, Z., Yokoyama, K.: Pulsefield magnetization for disc-shaped $\mathrm{MgB}_{2}$ bulk magnets. IEEE Trans. Appl. Supercond. 27, 6800504 (2017)

Publisher's Note Springer Nature remains neutral with regard to jurisdictional claims in published maps and institutional affiliations. 